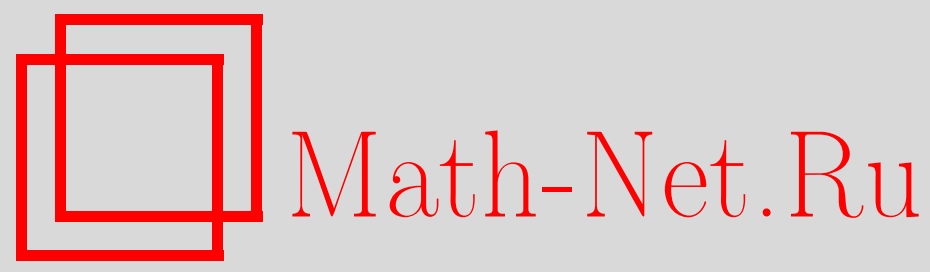

И. Д. Чуешов, Замечание о множествах определяющих элементов для систем реакции-диффузии, Матем. заметки, 1998, том 63, выпуск 5, 774-784

DOI: https://doi.org/10.4213/mzm1344

Использование Общероссийского математического портала Math-Net.Ru подразумевает, что вы прочитали и согласны с пользовательским соглашением http://www.mathnet.ru/rus/agreement

Параметры загрузки:

IP : 54.205 .225 .156

26 апреля 2023 г., $17: 32: 16$

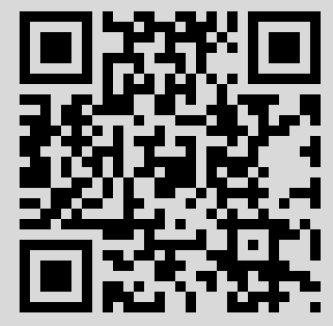




\section{ЗАМЕЧАНИЕ О МНОЖЕСТВАХ ОПРЕДЕЛЯЮЩИХ ЭЛЕМЕНТОВ ДЛЯ СИСТЕМ РЕАКЦИИ-ДИФФУЗИИ}

\section{И. Д. Чуешов}

Для некоторого класса систем уравнений параболического типа найдены условия, при которых конечное множество линейных функционалов, заданных на фазовом пространстве, однозначно определяет асимптотическое поведение решений. Указаны случаи, когда определяющие функционалы достаточно задавать лишш на части компонентов вектора состояния. В качестве примеров рассматриваются система уравнений, описьвающая реакцию Белоусова-Жаботинского, и двумерная система НавьеСтокса.

Библиография: 21 название.

1. Асимптотическое поведение бесконечномерных диссипативных систем во многих случаях может быть описано конечномерным глобальным аттрактором (см., например, [1]-[5] и ссылки, приведенные в этих работах). Однако детально исследовать структуру аттрактора удается лишь для весьма ограниченного класса задач. В этой связи важным является вопрос об отыскании минимальных (или близких к минимальньп) множеств естественных параметров задачи, которые однозначно определяют асимптотическое поведение системы. Впервые данньй вопрос рассматривался для двумерной системы Навье-Стокса в работах [6] и [7], где было показано, что асимптотическое поведение решений полностью определяется динамикой первых $N$ мод Фурье, если $N$ достаточно велико. После этих работ подобные результаты были получены для других параметров и уравнений (см., например, [8]-[10] и ссылки, приведенные в этих работах, а также в обзоре [4]). Были введены понятия определяющих узлов [11], [12] и определяющих локальных объемных средних [13], [14]. Обсуждался общий подход к проблеме существования конечного числа определяющих параметров [15].

В данной заметке рассматриваются системы параболических уравнений типа реакции-диффузии и найдены условия, при которых конечное множество линейных функционалов, заданных на фазовом пространстве, однозначно определяет асимптотическое поведение решений. Полученные результаты позволяют, в частности, для рассматриваемого класса систем доказать существование конечных наборов определяющих мод, узлов и локальных объемных средних. Оказалось также, что в некоторых случаях определяющие функционалы достаточно задавать лишь на части компонентов вектора состояния. В качестве примера рассматривается система уравнений, описьвающая реакцию Белоусова-Жаботинского.

Представленные методы носят общий характер. Они позволяют, например, для двумерной системы Навье-Стокса показать, что значения одного из компонентов вектора скорости в достаточно большом конечном множестве узлов однозначно определяют асимптотическое поведение решений. 
2. Предположим, что $\Omega$ - гладкая ограниченная область в $\mathbb{R}^{n}, n \geqslant 1, H^{s}(\Omega)$ - соболевское пространство порядка $s$ на $\Omega$ и $H_{0}^{s}(\Omega)$ - замыкание в $H^{s}(\Omega)$ множества бесконечно дифференцируемых функций с компактным носителем в $\Omega$. Пусть $\|\cdot\|_{s}-$ норма в $H^{s}(\Omega)$, а $\|\cdot\|$ и $(\cdot, \cdot)$ - норма и скалярное произведение в $L^{2}(\Omega)$. В дальнейшем используются также пространства

$$
\boldsymbol{H}^{s}=\left(H^{s}(\Omega)\right)^{m} \equiv H^{s}(\Omega) \times \cdots \times H^{s}(\Omega), \quad m \geqslant 1 .
$$

Аналогичньй смысл имеют обозначения $\boldsymbol{L}^{2}$ и $\boldsymbol{H}_{0}^{s}$. Нормы и скалярные произведения в $\boldsymbol{L}^{2}$ и $\boldsymbol{H}^{s}$ будем обозначать так же, как и в $L^{2}(\Omega)$ и $H^{s}(\Omega)$.

В качестве основной модели рассматривается следующая система уравнений:

$$
\begin{gathered}
\partial_{t} u=a(x, t) \Delta u-f(x, u, \nabla u ; t), \quad x \in \Omega, \quad t>0, \\
\left.u\right|_{\partial \Omega}=0, \quad u(x, 0)=u_{0}(x),
\end{gathered}
$$

где $u(x, t)=\left(u_{1}(x, t), \ldots, u_{m}(x, t)\right), \Delta$ - оператор Лапласа, $\nabla u_{k}=\left(\partial_{x_{1}} u_{k}, \ldots, \partial_{x_{n}} u_{k}\right)$, $a(x, t)$ - матрица порядка $m \times m$ с элементами из $L^{\infty}\left(\bar{\Omega} \times \mathbb{R}_{+}\right)$такая, что для всех $x \in \bar{\Omega}$ и $t \in \mathbb{R}_{+}$

$$
a_{+}(x, t)=\frac{1}{2}\left(a+a^{*}\right) \geqslant \mu_{0} I, \quad \mu_{0}>0 .
$$

Предполагается также, что непрерьвная функция

$$
f=\left(f_{1}, \ldots, f_{m}\right): \bar{\Omega} \times \mathbb{R}^{(n+1) m} \times \mathbb{R}_{+} \rightarrow \mathbb{R}^{m}
$$

такова, что у задачи (1) существуют решения, принадлежащие классу $\mathscr{W}$ функций на $\mathbb{R}_{+}$ со свойствами:

а) для любого $u \in \mathscr{W}$ существует $t_{0}>0$ такое, что

$$
u(t) \in C\left(t_{0},+\infty ; \boldsymbol{H}^{2} \cap \boldsymbol{H}_{0}^{1}\right), \quad \partial_{t} u(t) \in C\left(t_{0},+\infty ; \boldsymbol{L}^{2}\right)
$$

где $C(a, b ; X)$ - пространство сильно непрерьвных функций на $[a, b]$ со значениями в $X$;

б) существует константа $k>0$ такая, что для любых $u, v \in \mathscr{W}$ найдется $t_{1}>0$ такое, что при $t \geqslant t_{1}$ имеем

$$
\|f(u, \nabla u ; t)-f(v, \nabla v ; t)\| \leqslant k(\|u-v\|+\|\nabla u-\nabla v\|) .
$$

ЗАмЕчАниЕ 1 . Если $a(x, t)$ - диагональная матрица с элементами из $C^{2}\left(\bar{\Omega} \times \mathbb{R}_{+}\right)$, а $f$ - непрерьвно дифференцируемое отображение такое, что

$$
|f(x, u, p ; t)-f(x, v, q ; t)| \leqslant k(|u-v|+|p-q|)
$$

для всех $u, v \in \mathbb{R}^{m}, p, q \in \mathbb{R}^{n m}, x \in \bar{\Omega}$ и $t \in \mathbb{R}_{+}$, то при вьполнении естественных условий согласования задача (1) имеет единственное классическое решение [16], которое, очевидно, обладает свойствами (3) и (4). В том случае, когда динамическая система, 
отвечающая (1), является диссипативной, условие глобальной липшицевости (5) можно ослабить. Например (см. [1]), если $a$ - постоянная матрица и

$$
f(x, u, \nabla u ; t)=\bar{f}(x, u)+\sum_{j=1}^{n} b_{j}(x) \partial_{x_{j}} u+g(x),
$$

где $b_{j}=\left(b_{j}^{1}, \ldots, b_{j}^{m}\right) \in \boldsymbol{L}^{\infty}, g=\left(g_{1}, \ldots, g_{m}\right) \in \boldsymbol{L}^{2}$, a $\bar{f}=\left(\bar{f}_{1}, \ldots, \bar{f}_{m}\right)$ непрерывно дифференцируема и удовлетворяет условиям

$$
\begin{gathered}
\bar{f}(x, u) u \geqslant \mu_{1}|u|^{p_{0}}, \quad|\bar{f}(x, u)| \leqslant \mu_{2}|u|^{p_{0}-1}+C, \quad p_{0}>2, \\
\left|\frac{\partial \bar{f}_{k}}{\partial u_{j}}\right| \leqslant C\left(1+|u|^{p_{1}}\right), \quad 1 \leqslant k \leqslant m, \quad 1 \leqslant j \leqslant n,
\end{gathered}
$$

где $\mu_{1}, \mu_{2}>0$ и $p_{1} \leqslant \min \{4 / n, 2 /(n-2)\}$ при $n>2$, то любое решение задачи (1) с начальньг условием из $\boldsymbol{L}^{2}$ единственно и обладает свойствами $(3),(4)$.

ОПРЕДЕЛЕНИЕ 1 . Пусть $\mathscr{L}=\left\{l_{j}: j=1, \ldots, N\right\}$ - множество линейных функционалов на $\boldsymbol{H}^{2}$. Тогда $\mathscr{L}$ назьвается множеством определяющих әлементов задачи (1) в классе $\mathscr{W}$, если для любой пары ее решений $u, v \in \mathscr{W}$ из условия

$$
\lim _{t \rightarrow \infty}\left(l_{j}(u(t))-l_{j}(v(t))\right)=0 \quad \text { для } j=1, \ldots, N
$$

вытекает, что

$$
\lim _{t \rightarrow \infty}\|u(t)-v(t)\|=0
$$

Для характеризации множества определяющих элементов будем использовать следующее понятие дефекта полноты.

ОПРЕДЕЛЕНИЕ 2 . Пусть $V$ и $H$ - рефлексивные банаховыпространства, $V$ компактно и плотно вложено в $H$. Дефектом полноты множества $\mathscr{L}$ линейных функционалов на $V$ относительно $H$ назьвается величина

$$
\varepsilon \mathscr{L}(V, H)=\sup \left\{\|w\|_{H}: w \in V, l(w)=0, l \in \mathscr{L},\|w\|_{V} \leqslant 1\right\} .
$$

Нетрудно показать, что величина $\varepsilon \mathscr{L}(V, H)$ тесно связана с понятием аппроксимационной ошибки (см., например, [17], а также приведенные ниже примеры).

Сформулируем основное утверждение.

Теорема 1. Множество $\mathscr{L}=\left\{l_{j}: j=1, \ldots, N\right\}$ линейно независимых функиионалов на $\boldsymbol{H}^{2} \cap \boldsymbol{H}_{0}^{1}$ является мноэсеством определяющих әлементов задачи (1) в классе $\mathscr{W}$, если

$$
\varepsilon \mathscr{L}\left(\boldsymbol{H}^{2} \cap \boldsymbol{H}_{0}^{1}, \boldsymbol{L}^{2}\right)<\frac{c_{0} \mu_{0}}{k \sqrt{2}}\left(1+\frac{27}{4}\left(\frac{k}{\mu_{0}}\right)^{2}\right)^{-1 / 2} \equiv \rho\left(k, \mu_{0}\right),
$$

где $c_{0}^{-1}=\sup \left\{\|w\|_{2}: w \in\left(H^{2} \cap H_{0}^{1}\right)(\Omega),\|\Delta w\| \leqslant 1\right\}$, а $\mu_{0}$ uk-константы из $(2)$ и (4). Более того, при условии (9) соотношение (6) влечет асимптотическую близость решений и и в пространстве $\boldsymbol{H}^{1}$ :

$$
\lim _{t \rightarrow \infty}\|u(t)-v(t)\|_{1}=0 .
$$


ДокАЗАТЕЛЬСТво. Пусть $u, v \in \mathscr{W}$. Тогда из (1) для $w=u-v$ имеем

$$
\partial_{t} w=a(x, t) \Delta w-(f(x, u, \nabla u ; t)-f(x, v, \nabla v ; t)) .
$$

Умножая скалярно в $\boldsymbol{L}^{2}$ на $\Delta w$ и пользуясь (4), находим, что

$$
\frac{1}{2} \frac{d}{d t}\|\nabla w(t)\|^{2}+\mu_{0}\|\Delta w(t)\|^{2} \leqslant k(\|w(t)\|+\|\nabla w(t)\|)\|\Delta w(t)\|
$$

для достаточно больших $t>0$. Поэтому неравенство $\|\nabla w\|^{2}=|(w, \Delta w)| \leqslant\|w\| \cdot\|\Delta w\|$ позволяет получить оценку

$$
\frac{d}{d t}\|\nabla w\|^{2}+\mu_{0}\|\Delta w\|^{2} \leqslant \frac{2 k^{2}}{\mu_{0}}\left(1+\frac{27}{4}\left(\frac{k}{\mu_{0}}\right)^{2}\right)\|w\|^{2} .
$$

Для $w \in \boldsymbol{H}^{2} \cap \boldsymbol{H}_{0}^{1}$ положим $\psi=w-\sum_{j=1}^{N} l_{j}(w) e_{j}$, где $\left\{e_{j}\right\}-$ элементы из $\boldsymbol{H}^{2} \cap \boldsymbol{H}_{0}^{1}$ такие, что $l_{j}\left(e_{i}\right)=0$ при $j \neq i$ и $l_{j}\left(e_{j}\right)=1$. Из определения (8) имеем, что $\|\psi\| \leqslant \varepsilon \mathscr{L}\|\psi\|_{2}$, где $\varepsilon_{\mathscr{L}} \equiv \varepsilon_{\mathscr{L}}\left(\boldsymbol{H}^{2} \cap \boldsymbol{H}_{0}^{1}, \boldsymbol{L}^{2}\right)$. Поэтому

$$
\|w\|^{2} \leqslant C(N, \delta) \max _{j}\left|l_{j}(w)\right|^{2}+(1+\delta) \varepsilon_{\mathscr{L}}^{2}\|w\|_{2}^{2}
$$

для любого $\delta>0$. Следовательно, (11) дает, что

$$
\frac{d}{d t}\|\nabla w(t)\|^{2}+\mu_{0}\left(1-\frac{(1+\delta) \varepsilon_{\mathscr{L}}^{2}}{\rho\left(k, \mu_{0}\right)^{2}}\right)\|\Delta w(t)\|^{2} \leqslant C \max _{j}\left|l_{j}(w)\right|^{2}
$$

где $\rho\left(k, \mu_{0}\right)$ определено в соотношении (9). Отсюда при условии (9) находим. что существует $\beta>0$ такое, что

$$
\|\nabla w(t)\|^{2} \leqslant e^{-\beta\left(t-t_{0}\right)}\left\|\nabla w\left(t_{0}\right)\right\|^{2}+C \int_{t_{0}}^{t} e^{-\beta(t-\tau)} \max _{j}\left|l_{j}(w(\tau))\right|^{2} d \tau
$$

для всех $t \geqslant t_{0}$, где $t_{0}$ достаточно велико. Поэтому из (6) следует (7) и (10). Теорема доказана.

ЗАмЕчАниЕ 2. Предположим, что $u(t)$ и $v(t)$ - два решения уравнения (1), определенные для всех $t \in \mathbb{R}$. Пусть (3) и (4) выполнены для каждого $t_{0}$ и

$$
\sup _{t<0}(\|\nabla u(t)\|+\|\nabla v(t)\|)<\infty .
$$

Тогда в условиях теоремы 1 равенства $l_{j}(u(t))=l_{j}(v(t))$ при $j=1, \ldots, N$ и $t \leqslant s$ для некоторого $s \leqslant \infty$, а также соотношение (13) дают, что

$$
\|\nabla w(t)\|^{2} \leqslant e^{-\beta\left(t-t_{0}\right)}\left\|\nabla w\left(t_{0}\right)\right\|^{2}
$$

для всех $t \in\left[t_{0}, s\right)$. Поэтому, устремляя $t_{0} \mathrm{\kappa}-\infty$, с помощью (14) находим, что $w(t) \equiv 0$ для всех $t \leqslant s$. Таким образом, если выполнено (9), то решения, ограниченные на всей оси в пространстве $\boldsymbol{H}^{1}$, однозначно определяются своими значениями на функционалах $l_{j} \in \mathscr{L}$. Именно это свойство определяющих элементов было использовано в работе [7] в качестве определения определяющих мод для системы Навье-Стокса. 
3. Теорема 1 показывает, что для того чтобы $\mathscr{L}$ было множеством определяющих элементов достаточно, чтобы дефект полноты $\varepsilon \mathscr{L}$ был мал. Приведем несколько примеров, когда $\varepsilon \mathscr{L}$ оценить довольно просто.

ПРимеР 1 (определяющие моды, $m \geqslant 1$ ). Пусть $\left\{e_{k}\right\}$ - собственные элементы оператора $-\Delta$ в $\boldsymbol{L}^{2}$ с условиями Дирихле на $\partial \Omega$, и $0<\lambda_{1} \leqslant \lambda_{2} \leqslant \cdots-$ соответствующие собственные числа. Тогда для дефекта полноты множества

$$
\mathscr{L}=\left\{l_{j}: l_{j}(w)=\int_{\Omega} w(x) e_{j}(x) d x, j=1, \ldots, N\right\}
$$

легко получить оценку $\varepsilon \mathscr{L}\left(\boldsymbol{H}^{2} \cap \boldsymbol{H}_{0}^{1}, \boldsymbol{L}^{2}\right) \leqslant \sqrt{n} \lambda_{N+1}^{-1}$. Таким образом, если $N$ таково, что $\lambda_{N+1}>\sqrt{n} \rho\left(k, \mu_{0}\right)^{-1}$, то $\mathscr{L}$ является множеством определяющих элементов.

Дальнейшие примеры основаны на следуюших леммах.

ЛЕмма 1. Пусть функиия $\lambda(x) \in L^{\infty}\left(\mathbb{R}^{n}\right)$ имеет компактный носитель и

$$
\int_{\mathbb{R}^{n}} \lambda(x) d x=1
$$

Для $h>0$ определим на $H^{2}(\Omega)$ функичональ

$$
l_{j}(w)=\frac{1}{h^{n}} \int_{\Omega} w(x) \lambda\left(\frac{x}{h}-j\right) d x, \quad j \equiv\left(j_{1}, \ldots, j_{n}\right) \in \mathscr{J}
$$

əəe

$$
\mathscr{J} \equiv\left\{\left(j_{1}, \ldots, j_{n}\right) \in \mathbb{Z}^{n}:\left(j_{1} h, \ldots, j_{n} h\right) \in \Omega\right\} .
$$

Тогда для дефекта полноты мнохества $\mathscr{L}=\left\{l_{j}: j \in \mathscr{J}\right\}$ справедлива оченка $\varepsilon_{\mathscr{L}}\left(H^{2}(\Omega), L^{2}(\Omega)\right) \leqslant c h^{2}$, әде $c$ - некоторая константа, зависящая от $\lambda(x)$.

ДокАЗАТЕЛьСтво. Незначительная модификация рассуждений из [17] позволяет показать, что существует функция $\mu(x) \in H^{2}\left(\mathbb{R}^{n}\right)$ с компактным носителем такая, что $\left\|w-R_{h} w\right\| \leqslant C h^{2}\|w\|_{2}$, где оператор $R_{h}$ определяется формулой

$$
R_{h}(w)=\sum_{j \in D(h, \mu)} \frac{1}{h^{n}} \int_{\Omega} w(y) \lambda\left(\frac{y}{h}-j\right) d y \cdot \mu\left(\frac{x}{h}-j\right)
$$

Здесь

$$
D(h, \mu)=\left\{j \equiv\left(j_{1}, \ldots, j_{n}\right) \in \mathbb{Z}^{n}: \Omega \cap \operatorname{supp} \mu\left(\frac{x}{h}-j\right) \neq \varnothing\right\} .
$$

Поэтому искомая оценка вытекает из очевидного неравенства

$$
\varepsilon \mathscr{L}_{h} \leqslant \sup \left\{\left\|w-R_{h} w\right\|:\|w\|_{2} \leqslant 1\right\}
$$


Лемма 2. Предположим, что $n \leqslant 3$. Пусть $\mathscr{T}^{h}-$ триангуляиия (см. [18]) области $\Omega$, образованная тетраәдрами с ребрами не больше чем $h$, и пусть $\left\{x_{j}: j=\right.$ $\left.1, \ldots, N_{h}\right\}$ - вершины всех тетраәдров, образуюших триангуляиию $\mathscr{T}^{h}$. Тогда для множества функционалов

$$
\mathscr{L}=\left\{l_{j}: l_{j}(w)=w\left(x_{j}\right), j=1, \ldots, N_{h}\right\}
$$

справедлива оченка $\varepsilon \mathscr{L}\left(H^{2}(\Omega), L^{2}(\Omega)\right) \leqslant c h^{2}$, әде $c$ - некоторая абсолютная константа.

ДокАЗАТЕЛЬСТВО. Пусть $S\left(\mathscr{T}^{h}, w\right)$ - интерполящионньй линейньй сплайн функции $w \in H^{2}(\Omega)$, отвечающий триангулящии $\mathscr{T}^{h}$. Очевидно, что $l_{j}\left(S\left(\mathscr{T}^{h}, w\right)\right)=w\left(x_{j}\right)$. Поэтому, как и в предыдущей лемме, искомая оценка вытекает из соотношения (см. [18]) $\left\|w-S\left(\mathscr{T}^{h}, w\right)\right\| \leqslant C h^{2}\|w\|_{2}$.

Лемма 3. Пусть $V_{k} u H_{k}$ - рефлексивные банаховы пространства, $V_{k}$ компактно и плотно вложено в $H_{k}, \mathscr{L}_{k}$ - множсество линейных функиионалов на $V_{k}$, $k=1,2$. Положим $\mathscr{L}=\overline{\mathscr{L}}_{1} \cup \overline{\mathscr{L}}_{2} \subset\left(V_{1} \times V_{2}\right)^{*}$, әде

$$
\overline{\mathscr{L}}_{k}=\left\{l \in V^{*}: l\left(v_{1}, v_{2}\right)=l\left(v_{k}\right), l \in \mathscr{L}_{k}\right\}, \quad k=1,2 .
$$

Тогда $\varepsilon \mathscr{L}\left(V_{1} \times V_{2}, H_{1} \times H_{2}\right)=\max \left\{\varepsilon_{\mathscr{L}_{1}}\left(V_{1}, H_{1}\right), \varepsilon_{\mathscr{L}_{2}}\left(V_{2}, H_{2}\right)\right\}$.

Доказательство легко следует из определения 2.

Леммы 1-3 позволяют построить следующие примеры.

ПримеР 2 (определяющие локальные объемные средние с весом $\lambda$ ). Пусть $\mathscr{L}_{h}-$ множество функционалов на $H^{2}(\Omega)$, описанное в лемме 1 , и

$$
\mathscr{L}_{h}^{k}=\left\{l \in\left(\boldsymbol{H}^{2}\right)^{*}: l(w)=l\left(w_{k}\right), w \in \boldsymbol{H}^{2}, l \in \mathscr{L}_{h}\right\} .
$$

Тогда, если $h$ достаточно мало, то $\bigcup_{k=1}^{m} \mathscr{L}_{h}^{k}$ является множеством определяющих элементов задачи (1). Отметим, что если $\lambda(x)=1$ при $x \in S \equiv[0,1]^{n}$ и $\lambda(x)=0$ для $x \notin S$, то получатся локальные объемные средние (ср. [10], [14]).

ПримеР 3 (определяющие узлы $n \leqslant 3$ ). Пусть $\mathscr{L}_{h}^{k}$ определяются формулой $(16)$, где $\mathscr{L}_{h}$ имеет вид (15). Тогда $\bigcup_{k=1}^{m} \mathscr{L}_{h}^{k}$ является множеством определяющих элементов задачи (1) при условии, что $h$ достаточно мало.

Ясно также, что лемма 3 позволяет строить смешанные определяющие элементы: по одним компонентам вектора состояния это - определяющие узлы, по другим - локальные объемные средние с весом $\lambda$. Возможны и другие варианты.

4. Может оказаться, что не все компоненты вектора решений $u(x, t)$ будут существенньми для однозначного определения асимптотического поведения. Приведенная ниже теорема показьвает, когда такая ситуация может возникнуть.

Пусть $I$ - подмножество в $\{1, \ldots, m\}$. Введем пространства

$$
\boldsymbol{H}_{I}^{s}=\left\{w=\left(w_{1}, \ldots, w_{m}\right) \in \boldsymbol{H}^{s}: w_{k} \equiv 0, k \notin I\right\}, \quad s \in \mathbb{R} .
$$

Эти пространства будут отождествляться с $\left(H^{s}(\Omega)\right)^{|I|}$, где $|I|$ - число элементов множества $I$. Аналогичньй смысл имеют обозначения $\boldsymbol{L}_{I}^{2}$ и $\boldsymbol{H}_{0, I}^{s}$. Множество $\mathscr{L}$ линейных функционалов на $\boldsymbol{H}_{I}^{2}$ назьвается определяющ, $и$, если таковым является $p_{I}^{*} \mathscr{L}$, где $p_{I}-$ естественная проекция $\boldsymbol{H}^{s}$ на $\boldsymbol{H}_{I}^{s}$. 
Теорема 2. Пусть $a=\operatorname{diag}\left(d_{1}, \ldots, d_{m}\right)-$ диагональная матрииа с постоянными элементами, и пусть $\left\{I, I^{\prime}\right\}$ - разбиение множсества $\{1, \ldots, m\}$ на два непересекаюшихся подмножества. Предположим, что существуют положительные константы $\omega, k^{*}, \theta_{i}$, әде $i=1, \ldots, m$, такие, что для любой пары решений $u, v \in \mathscr{W}$ справедливо неравенство (ниже $w=u-v$ ):

$$
\begin{aligned}
& \sum_{i \in I} \theta_{i}\left(-\frac{d_{i}}{2}\left\|\Delta w_{i}\right\|^{2}+\left(f_{i}(u, \nabla u ; t)-f_{i}(v, \nabla v ; t), \Delta w_{i}\right)\right) \\
& +\sum_{i \in I^{\prime}} \theta_{i}\left(-d_{i}\left\|\nabla w_{i}\right\|^{2}-\left(f_{i}(u, \nabla u ; t)-f_{i}(v, \nabla v ; t), w_{i}\right)\right) \\
& \leqslant-\omega \sum_{i \in I^{\prime}}\left\|w_{i}\right\|^{2}+k^{*} \sum_{i \in I}\left\|w_{i}\right\|^{2}
\end{aligned}
$$

Тогда множсество $\mathscr{L}=\left\{l_{j}: j=1, \ldots, N\right\}$ линейно независимых функционалов на $\boldsymbol{H}_{I}^{2} \cap \boldsymbol{H}_{0, I}^{1}$ является мнохсеством определяюших әлементов задачи (1) в классе $\mathscr{W}$, если

$$
\varepsilon_{\mathscr{L}} \equiv \varepsilon_{\mathscr{L}}\left(\boldsymbol{H}_{I}^{2} \cap \boldsymbol{H}_{0, I}^{1}, \boldsymbol{L}_{I}^{2}\right)<c_{0} \min _{i \in I} \sqrt{\frac{d_{i} \theta_{i}}{2 k^{*}}},
$$

где $c_{0}>0$ определяется так жее, как и в (9). Более того, если два решения $u, v \in \mathscr{W}$ обладают свойством

$$
\lim _{t \rightarrow \infty}\left(l_{j}\left(p_{I} u(t)\right)-l_{j}\left(p_{I} v(t)\right)\right)=0 \quad \partial \Omega_{\Im} \quad j=1, \ldots, N
$$

где $p_{I}$ - естественная проекиия $\boldsymbol{H}^{s}$ на $\boldsymbol{H}_{I}^{s}$, то справедливо также (10).

ДокАЗАТЕЛЬСТво. Пусть $u, v \in \mathscr{W}$ и $w=u-v$. Тогда

$$
\partial_{t} w_{i}=d_{i} \Delta w_{i}-\left(f_{i}(x, u, \nabla u ; t)-f_{i}(x, v, \nabla v ; t)\right)
$$

при $i=1, \ldots, m$. Умножим уравнения (20) скалярно в $L^{2}(\Omega)$ на $-\theta_{i} \Delta w_{i}$ при $i \in I$ и на $\theta_{i} w_{i}$ при $i \in I^{\prime}$ и просуммируем. Пользуясь (17), находим, что

$$
\frac{1}{2} \frac{d}{d t} \Phi(w(t))+\frac{1}{2} \sum_{i \in I} d_{i} \theta_{i}\left\|\Delta w_{i}\right\|^{2}+\omega \sum_{i \in I^{\prime}}\left\|w_{i}\right\|^{2} \leqslant k^{*} \sum_{i \in I}\left\|w_{i}\right\|^{2}
$$

где

$$
\Phi(w)=\sum_{i \in I} \theta_{i}\left\|\nabla w_{i}\right\|^{2}+\sum_{i \in I^{\prime}} \theta_{i}\left\|w_{i}\right\|^{2} .
$$

Как и при доказательстве теоремы 1 (см. (12)), имеем

$$
\sum_{i \in I}\left\|w_{i}\right\|^{2} \leqslant C(N, \delta) \max _{j}\left|l_{j}\left(p_{I} w\right)\right|^{2}+\frac{1+\delta}{c_{0}^{2}} \varepsilon_{\mathscr{L}}^{2} \sum_{i \in I}\left\|\Delta w_{i}\right\|^{2}
$$

для каждого $\delta>0$. Поэтому при условии (18) имеем

$$
\frac{d}{d t} \Phi(w(t))+\beta \Phi(w(t)) \leqslant C \max _{j}\left|l_{j}\left(p_{I} w(t)\right)\right|^{2}
$$


с некоторой константой $\beta>0$. Отсюда при условии (19) находим, что $\Phi(w(t)) \rightarrow 0$, когда $t \rightarrow 0$. Следовательно, имеет место (7). Теперь, используя (11), легко получить (10).

В качестве приложения теоремы 2 рассмотрим систему уравнений, описьвающих реакцию Белоусова-Жаботинского. Эта система (см. [2], [19] и приведенные там ссылки) получается из (1), если положить $n \leqslant 3, m=3, a(x, t)=\operatorname{diag}\left(d_{1}, d_{2}, d_{3}\right)$ и $f(x, u, \nabla u ; t) \equiv f(u)$, где

$f_{1}(u)=-\alpha\left(u_{2}-u_{1} u_{2}+u_{1}-\beta u_{1}^{2}\right), \quad f_{2}(u)=-\frac{1}{\alpha}\left(\gamma u_{3}-u_{2}-u_{1} u_{2}\right), \quad f_{3}(u)=-\delta\left(u_{1}-u_{3}\right)$, $\alpha, \beta, \gamma, \delta$-положительные числа. Теорема существования классических решений может быть установлена без особого труда (см., например, [16]). Хорошо также известно [19], что если $a_{3}>a_{1}>\max \left\{1, \beta^{-1}\right\}$ и $a_{2}>\gamma a_{3}$, то область

$$
D=\left\{u \equiv\left(u_{1}, u_{2}, u_{3}\right): 0 \leqslant u_{j} \leqslant a_{j}, j=1,2,3\right\} \subset \mathbb{R}^{3}
$$

является инвариантной (если вектор начальных условий лежит в $D$ для всех $x \in \Omega$, то $u(x, t) \in D$ при $x \in \Omega, t>0)$. Пусть $\mathscr{W} \equiv \mathscr{W}_{D}-$ множество классических решений с начальными условиями, имеющими значения в $D$. Ясно, что для $\mathscr{W}$ вьполняются предположения (3) и (4). Простые вычисления показывают, что можно подобрать числа $\omega, k^{*}, \theta_{2}, \theta_{3}$ так, что соотношение (17) будет выполняться для $I=\{1\}, I^{\prime}=\{2,3\}$ с $\theta_{1}=1$. Поэтому, если $\mathscr{L}=\left\{l_{j}: j=1, \ldots, N\right\}$ - множество линейных функционалов на $H^{2}(\Omega) \cap H_{0}^{1}(\Omega)$ такое, что $\varepsilon_{\mathscr{L}}\left(\left(H^{2} \cap H_{0}^{1}\right)(\Omega), L^{2}(\Omega)\right)$ достаточно мало, то из условия

$$
\lim _{t \rightarrow \infty}\left(l_{j}\left(u_{1}(t)\right)-l_{j}\left(v_{1}(t)\right)\right)=0, \quad j=1, \ldots, N,
$$

для некоторой пары решений $u(t)=\left(u_{1}(t), u_{2}(t), u_{3}(t)\right)$ и $v(t)=\left(v_{1}(t), v_{2}(t), v_{3}(t)\right)$ из $\mathscr{W}$ вытекает, что $\|u(t)-v(t)\|_{1} \rightarrow 0$ при $t \rightarrow \infty$.

5. Представленньй выше подход может быть использован и при изучении системы Навье-Стокса. В качестве примера рассмотрим уравнения, описывающие динамику вязкой несжимаемой жидкости, заполняющей область $\Omega \equiv T^{2}=(0, L) \times(0, L)$, с периодическими граничными условиями:

$$
\begin{gathered}
\partial_{t} u-\nu \Delta u+(u, \nabla) u+\nabla p=F(x, t), \quad x \in T^{2}, \quad t>0, \\
\nabla u=0, \quad x \in T^{2}, \quad u(x, 0)=u_{0}(x),
\end{gathered}
$$

где неизвестные вектор скорости $u(x, t)=\left(u_{1}(x, t), u_{2}(x, t)\right)$ и давление $p(x, t)$ являются $L$-периодическими функциями по пространственным переменным, $\nu>0, F(x, t)-$ внешняя сила.

Введем некоторые определения. Пусть $\mathscr{V}$ - пространство тригонометрических полиномов $v(x)$ периода $L$ со значениями в $\mathbb{R}^{2}$ таких, что $\operatorname{div} v=0$ и $\int_{T^{2}} v(x) d x=0$. Пусть $H$ - замькание $\mathscr{V}$ в $\boldsymbol{L}^{2}, \Pi$ - ортогональньй проектор в $\boldsymbol{L}^{2}$ на $H, A=-\Pi \Delta u=-\Delta u$, $B(u, v)=\Pi(u, \nabla) v$ для всех $u$ и $v$ из $D(A)=H \cap \boldsymbol{H}^{2}$. Напомним (см. [20], [21]), что $A$ - положительньй оператор с дискретным спектром, а билинейньй оператор $B(u, v)$, дает непрерьвное отображение из $D(A) \times D(A)$ в $H$. Задача $(21)$ при этом запишется в виде

$$
\partial_{t} u+\nu A u+B(u, u)=\Pi F(t), \quad u_{\mid t=0}=u_{0} \in H .
$$

Хорошо известно (см., например, [20], [21]), что если $u_{0} \in H$ и $\Pi F(t) \in L^{\infty}\left(\mathbb{R}_{+} ; H\right)$, то у задачи $(22)$ существует единственное решение $u(t)$ такое, что

$$
u(t) \in C\left(\mathbb{R}_{+} ; H\right) \cap C\left(t_{0},+\infty ; D(A)\right), \quad t_{0}>0 .
$$


Лемма 4. Пусть $u, v \in D(A) u w=u-v$. Тогдa

$$
\begin{aligned}
& |(B(u, u)-B(v, v), A w)| \leqslant \sqrt{2}\|w\|_{\infty} \cdot\|\nabla w\| \cdot\|A u\|, \\
& |(B(u, u)-B(v, v), A w)| \leqslant c L\left\|\nabla w_{k}\right\| \cdot\|A w\| \cdot\|A u\|,
\end{aligned}
$$

әде $\|\cdot\|_{\infty}$ есть $L^{\infty}$-норма, $w_{k}-k$-й компонент вектора $w, k=1,2, c$ - некоторая абсолютная константа.

ДокАЗАТЕЛЬСТво. Используя тождество (см. [21])

$$
(B(u, w), A w)+(B(w, u), A w)+(B(w, w), A u)=0
$$

для $u, w \in D(A)$, легко обнаружить, что $(B(u, u)-B(v, v), A w)=-(B(w, w), A u)$. Поэтому достаточно оценить норму $\|(B(w, w) \|$. Условие несжимаемости $\nabla w=0$ дает, что

$$
(w, \nabla) w=\left(-w_{1} \partial_{2} w_{2}+w_{2} \partial_{2} w_{1}, w_{1} \partial_{1} w_{2}-w_{2} \partial_{1} w_{1}\right)
$$

Следовательно,

$$
\|(w, \nabla) w\|^{2} \leqslant 2\left(\left\|w_{1} \nabla w_{2}\right\|^{2}+\left\|w_{2} \nabla w_{1}\right\|^{2}\right) .
$$

Отсюда получаем (24). Докажем (25). Пусть для определенности $k=1$. Тогда (26) дает, что

$$
\|(w, \nabla) w\| \leqslant \sqrt{2}\left(\left\|w_{1}\right\|_{L^{4}} \cdot\left\|\nabla w_{2}\right\|_{L^{4}}+\left\|w_{2}\right\|_{\infty} \cdot\left\|\nabla w_{1}\right\|\right)
$$

Поэтому оценка (25) при $k=1$ вытекает из неравенств

$$
\|v\|_{L^{4}} \leqslant c_{1} L^{1 / 2}\|\nabla v\|, \quad\|v\|_{\infty} \leqslant c_{2} L\|\Delta v\|
$$

справедливых в рассматриваемом классе функций (см. [20], [21], а также работу [11], содержащую явные выражения для констант $c_{1}$ и $\left.c_{2}\right)$.

Теорема 3. 1. Множество $\mathscr{L}=\left\{l_{j}: j=1, \ldots, N\right\}$ линейно независимых функиионалов на $D(A)=\boldsymbol{H}^{2} \cap H$ является множеством определяюших әлементов задачи (22) в классе решений, обладающих свойством (23), если

$$
\varepsilon_{\mathscr{L}} \equiv \varepsilon_{\mathscr{L}}(D(A), H)<c_{1} G \equiv c_{1} \nu^{2}\left(\varlimsup_{t \rightarrow \infty}\|\Pi F(t)\|\right)^{-1}
$$

где $c_{1}-$ абсолютная константа.

2. Пусть $\mathscr{L}=\left\{l_{j}: j=1, \ldots, N\right\}$-множество линейных функиионалов на $H^{2}(\Omega)$ $u \varepsilon_{\mathscr{L}}^{\prime} \equiv \varepsilon_{\mathscr{L}}\left(H^{2}(\Omega), L^{2}(\Omega)\right)<c_{2} L^{-2} G^{2}$, где $c_{2}$ - некоторая абсолютная константа. Тогда кахдое из мнохеств $p_{k}^{*} \mathscr{L}$ является мнохсеством определяюших әлементов задачи (22) в классе решений со свойством (23). Здесь $p_{k}$ - естественная проекиия на $k$-й компонент вектора скорости, $p_{k}\left(u_{1}, u_{2}\right)=u_{k}, k=1,2$. 
ДокАЗАТЕЛЬСтво. Пусть $u(t), v(t)$ - решения задачи $(22)$, обладающие свойством (23). Тогда из (22) и (24) для $w=u-v$ имеем, что

$$
\frac{1}{2} \frac{d}{d t}\|\nabla w\|^{2}+\nu\|\Delta w\|^{2} \leqslant \sqrt{2}\|w\|_{\infty} \cdot\|\nabla w\| \cdot\|A u\| .
$$

Как и при доказательстве теоремы 1 (см. (12)), имеем оценку $\|w\| \leqslant C \eta(w)+\varepsilon \mathscr{L}\|\Delta w\|$, где $\eta(w)=\max _{j}\left|l_{j}(w)\right|$. Поэтому

$$
\|w\|_{\infty} \leqslant c_{0}\|w\|^{1 / 2} \cdot\|\Delta w\|^{1 / 2} \leqslant c_{1} \sqrt{\eta(w)}\|\Delta w\|^{1 / 2}+c_{0} \sqrt{\varepsilon \mathscr{L}}\|\Delta w\| .
$$

Неравенство $\|\nabla w\|^{2} \leqslant\|w\| \cdot\|\Delta w\|$ позволяет получить оценку $\varepsilon \mathscr{L}\left(D(A), D\left(A^{1 / 2}\right)\right) \leqslant \varepsilon_{\mathscr{L}}^{1 / 2}$ и, следовательно,

$$
\|\Delta w\|^{2} \geqslant-C_{\delta} \eta(w)^{2}+(1-\delta) \varepsilon_{\mathscr{L}}^{-1}\|\nabla w\|^{2}
$$

для каждого $\delta>0$. Поэтому, пользуясь свойствами диссипативности решений (см. [1], $[2])$, находим, что

$$
\frac{d}{d t}\|\nabla w(t)\|^{2}+\alpha(t)\|\nabla w\|^{2} \leqslant C\left(\eta(w)^{2}+\sqrt{\eta(w)}\right)
$$

где

$$
\alpha(t)=\nu(1-\delta) \varepsilon_{\mathscr{L}}^{-1}-2 c_{0}^{2} \varepsilon \mathscr{L} \nu^{-1}\|A u(t)\|^{2} .
$$

Отсюда, как и в работах [10], [14], используя некоторую модификацию лемму Гронуолла (см. лемму 4.1 в [14]), легко заключить, что в условиях теоремы имеется сходимость $\|\nabla w\| \rightarrow 0$.

Для того чтобы доказать вторую часть теоремы, воспользуемся аналогичными рассуждениями. Для определенности ограничимся случаем $k=1$. Из (22) и (25) имеем

$$
\frac{d}{d t}\|\nabla w\|^{2}+\nu\|\Delta w\|^{2} \leqslant c^{2} L^{2} \nu^{-1}\left\|\nabla w_{1}\right\|^{2} \cdot\|A u\|^{2}
$$

Отсюда, рассуждая как и вьше, находим, что

$$
\frac{d}{d t}\|\nabla w(t)\|^{2}+\alpha_{1}(t)\|\nabla w\|^{2} \leqslant C \eta(w)^{2}
$$

где

$$
\alpha_{1}(t)=\min \left\{\frac{\nu(1-\delta)}{\varepsilon_{\mathscr{L}}^{\prime}}-\frac{c^{2} L^{2}}{\nu}\|A u(t)\|^{2}, \nu\left(\frac{2 \pi}{L}\right)^{2}\right\} .
$$

Поэтому, как и выше, метод, представленньй в [10], [14], позволяет получить второе утверждение теоремы 3.

ЗАмечАниЕ 3. Различные варианты утверждения 1 теоремы 3 хорошо известны (см. [6], [10]-[14]) в случае, когда $\mathscr{L}$ одно из множеств, описанных в примерах 1-3. Для общей ситуации близкое утверждение в несколько иной форме было анонсировано в [15]. Приведенное здесь доказательство фактически повторяет рассуждение, использованное в [10] при построении конечного семейства определяющих узлов. Отметим также, что утверждение 1 и леммы 1,2 позволяют получить оценки для числа определяющих узлов и локальных объемных средних того же вида, что и в [10]. В то время как утверждение 2 , хотя и использует только один из компонентов вектора скорости, приводит, вообще говоря, к необходимости рассматривать значительно большее число определяющих функционалов по сравнению со случаем утверждения 1. 


\section{СПИСОК ЦИТИРОВАННОЙ ЛИТЕРАТУРЫ}

[1] Бабин А. В., Вишик М.И.Аттракторы эволюционных уравнений. М.: Наука, 1989.

[2] Temam R. Infinite-Dimensional Dynamical Systems in Mechanics and Physics. New York: Springer, 1988.

[3] Ладыженская О. А. О нахождении минимальных глобальных аттракторов для уравнения Навье-Стокса и других уравнений с частными производными // УМН. 1987. Т. 42. №6. C. $25-60$.

[4] Чуешов И. Д. Глобальные аттракторы в нелинейных задачах математической физики // УМH. 1993. Т. 48. №3. С. 135-162.

[5] Chepyzhov V.V., Vishik M.I. Attractors of non-autonomous dynamical systems and their dimension // J. Math. Pures Appl. (9). 1994. V. 73. P. 279-333.

[6] Foias C., Prodi G. Sur le comportement global des solutions non-stationnaires des équations de Navier-Stokes en dimension 2 // Rend. Sem. Mat. Univ. Padova. 1967. V. 39. P. 1-34.

[7] Ладыженская О.А. О динамической системе, порождаемой уравнениями Навье-Стокса // Записки научн. семин. ЛОМИ. 1972. Т. 27. С. 91-115.

[8] Ладыженская О.А. Об оценках фрактальной размерности и числа определяющих мод для инвариантных множеств динамических систем // Записки научн. семин. ЛОМИ. 1987. T. 163 . C. $105-129$.

[9] Foias C., Kukavica I. Determining nodes for the Kuramoto-Sivashinsky equation // J. Dynam. Differential Equations. 1995. V. 7. P. 365-373.

[10] Jones D. A., Titi E.S. Upper bounds on the number of determining modes, nodes and volume elements for the Navier-Stokes equations // Indiana Univ. Math. J. 1993. V. 42. P. 875-887.

[11] Foias C., Manley O.P., Temam R., Treve Y.M. Asymptotic analysis of the Navier-Stokes equations // Phys. D. 1983. V. 9. P. 157-188.

[12] Foias C., Temam R. Determination of solutions of the Navier-Stokes equations by a set of nodal values // Math. Comp. 1984. V. 43. P. 117-133.

[13] Foias C., Titi E. S. Determining nodes, finite difference schemes and inertial manifolds // Nonlinearity. 1991. V. 4. P. 135-153.

[14] Jones D.A., Titi E.S. Determination of the solutions of the Navier-Stokes equations by finite volume elements // Phys. D. 1992. V. 60. P. 165-174.

[15] Cockburn B., Jones D. A., Titi E.S. Determining degrees of freedom for nonlinear dissipative systems // C. R. Acad. Sci. Paris. Sér. I. Math. 1995. V. 321. P. 563-568.

[16] Ладыженская О. А., Солонников В. А., Уральцева Н. Н. Линейные и квазилинейные уравнения параболического типа. М.: Наука, 1967.

[17] Aubin J.-P. Approximation of Elliptic Boundary-Value Problems. New York: Wiley, 1972.

[18] Ciarlet P. The Finite Elements Method for Elliptic Problems. Amsterdam: North-Holland, 1978.

[19] Smoller J. Shock Waves and Reaction-Diffusion Equations. New York: Springer, 1983.

[20] Ладыженская О. А. Математические вопросы динамики вязкой несжимаемой жидкости. M.: Наука, 1970.

[21] Constantin P., Foias C. Navier-Stokes Equations. Chicago: Univ. of Chicago, 1988.

Харьковский государственный университет

E-mail: chueshov@ilt.kharkov.ua 